TRANSACTIONS OF THE

AMERICAN MATHEMATICAL SOCIETY

Volume 363, Number 1, January 2011, Pages 185-201

S 0002-9947(2010)04893-7

Article electronically published on August 24, 2010

\title{
GEVREY SOLVABILITY AND GEVREY REGULARITY IN DIFFERENTIAL COMPLEXES ASSOCIATED TO LOCALLY INTEGRABLE STRUCTURES
}

\author{
PAULO A. S. CAETANO AND PAULO D. CORDARO
}

\begin{abstract}
In this work we study some properties of the differential complex associated to a locally integrable (involutive) structure acting on forms with Gevrey coefficients. Among other results we prove that, for such complexes, Gevrey solvability follows from smooth solvability under the sole assumption of a regularity condition. As a consequence we obtain the proof of the Gevrey solvability for a first order linear PDE with real-analytic coefficients satisfying the Nirenberg-Treves condition $(\mathcal{P})$.
\end{abstract}

\section{INTRODUCTION}

Associated to any involutive structure over a smooth manifold (that is, a complex subbundle of its complexified tangent bundle satisfying the bracket condition) there is canonically associated a complex of (first order) differential operators (cf. T2, p. 32]). For instance, in the classical cases of real, complex and CR structures, this association gives, respectively, the well-known de Rham, Dolbeault and the $\bar{\partial}_{b}$ complexes, for which the study of solvability has already achieved the status of classical questions in the theory of linear PDEs and multi-dimensional complex analysis.

Within this framework a natural question is then the local Gevrey solvability for the differential complex associated to an arbitrary Gevrey locally integrable structure. A pioneering study in this direction can be found in Mi], where the author considers, via $L^{2}$-weighted estimates, the (micro-local) Gevrey solvability for the $\bar{\partial}_{b}$-complex on hypersurfaces in $\mathbb{C}^{n}$.

In the present work we return to this question but adopt a more abstract view: our main hypothesis is that, for some fixed degree, (weak)-classical solvability occurs and from this we conclude that Gevrey solvability is also true (cf. Theorem 5.1 below). For the proof we use two main tools: the ultra-differential operators of class $\left(p !^{s}\right)$ introduced by $\mathrm{H}$. Komatsu $\mathrm{KO}$ in conjunction with a notion of Gevrey classes within the concept of a hypo-analytic structure, which we introduce in Section 2.

As a consequence of this result we prove that, for any $s>1$, any first order linear PDE with analytic coefficients $P u=f$ satisfying the Nirenberg-Treves condition

Received by the editors November 19, 2007 and, in revised form, July 26, 2008.

2000 Mathematics Subject Classification. Primary 35A07; Secondary 35D10, 35N10.

Key words and phrases. Local solvability, Gevrey classes, locally integrable structures, hypoanalytic structures.

This research was partially supported by CNPq and Fapesp.

(C)2010 American Mathematical Society Reverts to public domain 28 years from publication 
$(\mathcal{P})$ has local solutions $u \in G^{s}$ for every $f \in G^{s}$, a result that we believe is new (see also [L] and Remark 6.1 below).

Also, as a byproduct of the tools we have developed in order to prove Theorem 5.1 , we obtain a representation formula for closed (in the sense of the differential complex under study) currents and, in the final section, a sufficient condition for Gevrey hypoellipticity for zero degree currents. This latter result can be considered as a preliminary step of the study of further questions to which we hope to return.

We emphasize that all results we obtain are consequences of a solvability and of a regularity result proved for a class of ultra-differentiable operators of class $\left(p !^{!}\right)$ that are elliptic in a very strong sense (cf. Definition 3.1 and Propositions 3.1 and 3.2 ). The main aspect of these operators is that they commute with our differential complex, a property that simplifies the arguments very much.

This paper is an extension of [C] and its roots lay in the work [Co, where a class of infinite order operators introduced by Kaneko $\mathrm{Ka}$. was adapted in order to obtain representation formulas for hyperfunction solutions in hypo-analytic structures.

Finally, we wish to thank Professor F. Treves for his suggestions and interest in this work.

\section{Preliminaries: Gevrey hypo-Analytic structures}

Let $\Omega$ be an open subset of $\mathbb{R}^{N}$. A (corank zero) hypo-analytic structure on $\Omega$ (cf. [T2]) is a collection of ordered pairs $\mathfrak{T}=\{(U, Z)\}$, where $U \subset \Omega$ is an open subset of $\Omega$ and $Z=\left(Z_{1}, \ldots, Z_{N}\right): U \longrightarrow \mathbb{C}^{N}$ is a smooth mapping, such that the following conditions are satisfied:

(1) $\Omega=\bigcup_{(U, Z) \in \mathfrak{T}} U$.

(2) If $(U, Z) \in \mathfrak{T}$, then the differentials $\mathrm{d} Z_{1}, \ldots, \mathrm{d} Z_{N}$ are $\mathbb{C}$-linearly independent at every point of $U$.

(3) If $(U, Z),\left(U^{\prime}, Z^{\prime}\right) \in \mathfrak{T}$ are such that $U \cap U^{\prime} \neq \emptyset$, then $(U, Z)$ and $\left(U^{\prime}, Z^{\prime}\right)$ are biholomorphically related; that is, there are open subsets $\mathcal{U} \supset Z\left(U \cap U^{\prime}\right)$, $\mathcal{U}^{\prime} \supset Z^{\prime}\left(U \cap U^{\prime}\right)$ of $\mathbb{C}^{N}$ and a biholomorphism $H: \mathcal{U} \rightarrow \mathcal{U}^{\prime}$ such that $Z^{\prime}=H \circ Z$ in $U \cap U^{\prime}$.

(4) If $U^{b}$ is an open subset of $\Omega$ and $Z^{b}=\left(Z_{1}^{b}, \ldots, Z_{N}^{b}\right): U^{b} \longrightarrow \mathbb{C}^{N}$ is a smooth mapping such that

- $\mathrm{d} Z_{1}^{b}, \ldots, \mathrm{d} Z_{N}^{b}$ are $\mathbb{C}$-linearly independent at every point of $U^{b}$,

- $\left(U^{\mathrm{b}}, Z^{\mathrm{b}}\right)$ and $(U, Z)$ are biholomorphically related for every $(U, Z) \in \mathfrak{T}$ with $U \cap U^{b} \neq \emptyset$,

then $\left(U^{b}, Z^{b}\right) \in \mathfrak{T}$.

Any pair $(U, Z) \in \mathfrak{T}$ is called a hypo-analytic chart related to $\mathfrak{T}$.

If $\Omega^{\prime} \subset \Omega$ is open, then the family $\left.\mathfrak{T}\right|_{\Omega^{\prime}} \doteq\left\{\left(U \cap \Omega^{\prime},\left.Z\right|_{U \cap \Omega^{\prime}}\right):(Z, U) \in \mathfrak{T}\right\}$ defines a hypo-analytic structure on $\Omega^{\prime}$, called the hypo-analytic structure on $\Omega^{\prime}$ induced by $\mathfrak{T}$.

Let $\Omega^{\prime} \subset \Omega$ be open. A function $u \in \mathcal{C}^{\infty}\left(\Omega^{\prime}\right)$ is said to be $\mathfrak{T}$-hypo-analytic if given any point $p \in \Omega^{\prime}$ and given any hypo-analytic chart $(U, Z) \in \mathfrak{T}$ such that $p \in U$, then $u=H \circ Z$ near $p$, where $H$ is a holomorphic map near $Z(p)$. We shall denote by $\mathcal{A}_{\mathfrak{T}}\left(\Omega^{\prime}\right)$ the space of all $\mathfrak{T}$-hypo-analytic functions on $\Omega^{\prime}$. It is clear that $\Omega^{\prime} \mapsto \mathcal{A}_{\mathfrak{T}}\left(\Omega^{\prime}\right)$ defines a sheaf, called the sheaf of germs of $\mathfrak{T}$-hypo-analytic functions on $\Omega$.

It is easily seen that any family $\mathfrak{T}_{0}=\{(U, Z)\}$ satisfying properties (1), (2) and (3) can be enlarged $\mathfrak{T}_{0} \subset \mathfrak{T}$ to a uniquely defined hypo-analytic structure on $\Omega$. 
In the following we assume given a hypo-analytic structure $\mathfrak{T}$ on $\Omega$.

Let $(U, Z) \in \mathfrak{T}$. We introduce a set of $N$ linearly independent, pairwise commuting complex vector fields $\mathrm{M}_{1}, \ldots, \mathrm{M}_{N}$ on $U$, defined by the relations

$$
\mathrm{d} Z_{k}\left(\mathrm{M}_{j}\right)=\mathrm{M}_{j} Z_{k}=\delta_{j k} .
$$

Given $V \Subset U, s \geq 1$ and $h>0$ we shall denote by $G^{s, h}(\bar{V} ; \mathrm{M})$ the space of all $f \in \mathcal{C}^{\infty}(\bar{V})$ such that, for some $C>0$,

$$
\sup _{\bar{V}}\left|\mathrm{M}^{\alpha} f\right| \leq C h^{|\alpha|}|\alpha| !^{s}, \quad \alpha \in \mathbb{Z}_{+}^{N} .
$$

Here, of course, we are setting $\mathrm{M}^{\alpha}=\mathrm{M}_{1}^{\alpha_{1}} \ldots \mathrm{M}_{N}^{\alpha_{N}}$.

The infimum of the constants $C$ for which (11) is valid defines a norm on $G^{s, h}(\bar{V} ; \mathrm{M})$ which turns it into a Banach space.

Example 2.1. If $\left(x_{1}, \ldots, x_{N}\right)$ denote the coordinates in $\mathbb{R}^{N}$ and if $Z$ denotes the inclusion map $\mathbb{R}^{N} \hookrightarrow \mathbb{C}^{N}$, then the corresponding vector fields for the hypo-analytic chart $\left(\mathbb{R}^{N}, Z\right)$ are $\partial / \partial x_{j}, j=1, \ldots, N$. Hence the associated spaces, for $V \Subset \mathbb{R}^{N}$, are the standard Gevrey spaces $G^{s, h}(\bar{V}, \mathrm{M})=G^{s, h}(\bar{V})$.

Before we proceed we just state a result whose proof can be easily adapted from an argument in ([Ko, p. 41]).

Proposition 2.1. Let $(U, Z) \in \mathfrak{T}$. Then given $V \Subset U$ with smooth boundary, $s \geq 1$ and $h<h^{\prime}$ the inclusion

$$
G^{s, h}(\bar{V} ; \mathrm{M}) \hookrightarrow G^{s, h^{\prime}}(\bar{V} ; \mathrm{M})
$$

is compact.

We now introduce the main object of the present study.

Definition 2.1. Let $s \geq 1$. A (corank zero) Gevrey hypo-analytic structure of order $s$ on $\Omega$ is a hypo-analytic structure $\mathfrak{T}$ on $\Omega$ such that, for any hypo-analytic chart $(U, Z) \in \mathfrak{T}$, the mapping $Z: U \rightarrow \mathbb{C}^{N}$ is Gevrey of order $s$.

If $\mathfrak{T}$ is a Gevrey hypo-analytic structure of order one, then $\mathfrak{T}$ is nothing else than the standard real-analytic structure on $\Omega$. If otherwise for some $(U, Z) \in \mathfrak{T}$ we have $Z$ real-analytic, then $\left.\mathfrak{T}\right|_{U}$ is the standard real-analytic structure on $U$. We also have

Proposition 2.2. Let $\mathfrak{T}$ be a Gevrey hypo-analytic structure of order $s$ on $\Omega$. Then for any open subset $\Omega^{\prime}$ of $\Omega$, the standard Gevrey space $G^{s}\left(\Omega^{\prime}\right)$ equals the space of all $f \in \mathcal{C}^{\infty}\left(\Omega^{\prime}\right)$ such that, for all $V \Subset \Omega^{\prime}$ with compact closure contained in the domain of a hypo-analytic chart $(U, Z) \in \mathfrak{T}$, there exists $h>0$ such that $\left.f\right|_{\bar{V}} \in G^{s, h}(\bar{V} ; \mathrm{M})$.

The proof follows simply from the fact that, in this case, each $\mathrm{M}_{j}$ has Gevrey coefficients of order $s$ (cf. [Ro]).

\section{UltradiffEREntial OPERATORS OF CLASS $\left(p !^{s}\right)$}

Let $P(\zeta)=\sum_{\alpha \geq 0} a_{\alpha} \zeta^{\alpha}$ be an entire function in $\mathbb{C}^{N}$. According to [Ko] the function $P(\zeta)$ defines an ultradifferential operator of class $\left(p !^{s}\right), s>1$, if there are constants $C>0, L>0$ such that

$$
\left|a_{\alpha}\right| \leq C L^{|\alpha|} /|\alpha| !^{s}
$$


If $(U, Z)$ is a hypo-analytic chart belonging to some hypo-analytic structure, we associate to each such $P(\zeta)$ an operator $P(\mathrm{M})$ defined, at least formally, by the expression

$$
P(\mathrm{M})=\sum_{\alpha \geq 0} a_{\alpha} \mathrm{M}^{\alpha} .
$$

Lemma 3.1. Let $\mathfrak{T}$ be a hypo-analytic structure on $\Omega$, let $(U, Z) \in \mathfrak{T}$, let $P(\zeta)$ define an ultradifferential operator of class $\left(p !^{s}\right)$ as above and let $h>0$. Then if $L \leq 1 /\left(2^{s+1} h\right)$, we have a continuous linear map

$$
P(\mathrm{M}): G^{s, h}(\bar{V} ; \mathrm{M}) \longrightarrow G^{s, 2^{s} h}(\bar{V} ; \mathrm{M}),
$$

for any $V \Subset U$.

Proof. Let $f \in G^{s, h}(\bar{V} ; \mathrm{M})$ satisfy (11). With $P(\zeta)$ as above we have

$$
\mathrm{M}^{\beta} P(\mathrm{M}) f=\sum_{\gamma \geq 0} a_{\gamma} \mathrm{M}^{\gamma+\beta} f .
$$

Hence, in $\bar{V}$ we can estimate

$$
\begin{aligned}
\left|\mathrm{M}^{\beta} P(\mathrm{M}) f\right| & \leq C \sum_{\gamma \geq 0} L^{|\gamma|} h^{|\gamma|+|\beta|}|\gamma| !^{-s}(|\gamma|+|\beta|) !^{s} \\
& =C h^{|\beta|}|\beta| !^{s} \sum_{\gamma \geq 0} L^{|\gamma|} h^{|\gamma|}\left(|\gamma| !^{-s}(|\gamma|+|\beta|) !^{s}\right) /|\beta| !^{s} \\
& \leq C\left(2^{s} h\right)^{|\beta|}|\beta| !^{s} \sum_{\gamma \geq 0}\left(2^{s} L h\right)^{|\gamma|} .
\end{aligned}
$$

Remark 3.1. If $P(\zeta)$ defines an ultradifferential operator of class $\left(p !^{s}\right)$, then the same is true for $P_{r}(\zeta)=P(r \zeta), r>0$. Moreover $P_{r}(\zeta)$ satisfies (2) for $r L$ substituted for $L$.

If $P(\zeta)$ defines an ultradifferential operator of class $\left(p !^{s}\right)$, then (2) implies the following property, for any $1 \leq s^{\prime}<s$ : given $\epsilon>0$ there is $C>0$ such that

$$
\left|a_{\alpha}\right| \leq C \epsilon^{|\alpha|} /|\alpha| !^{s^{\prime}}, \quad \alpha \in \mathbb{Z}_{+}^{N} .
$$

In particular, applying (3) when $s^{\prime}=1$ gives the following result:

Lemma 3.2. Let $\mathfrak{T}$ be a hypo-analytic structure on $\Omega$. Let $(U, Z) \in \mathfrak{T}$ and let $P(\zeta)$ define an ultradifferential operator of class $\left(p !^{s}\right)$, with $s>1$. Then the operator $P(\mathrm{M})$ defines an endomorphism of $\mathcal{A}_{\mathfrak{T}}(U)$.

Property (3) also allows us to extend the action of $P(\mathrm{M})$ to a much larger space in a more restricted situation.

We assume that $\mathfrak{T}$ is a Gevrey hypo-analytic structure of order $s_{0}>1$ and let $P(\zeta)$ define an ultradifferential operator of class $\left(p !^{s}\right)$ with $s>s_{0}$. Thanks to property (3) applied with $s_{0}$ substituted for $s^{\prime}$ it is easily seen that, for any $V \subset U$ open, there are well-defined endomorphisms $P(\mathrm{M}): G^{s_{0}}(V) \longrightarrow G^{s_{0}}(V)$, $P(\mathrm{M}): G_{c}^{s_{0}}(V) \longrightarrow G_{c}^{s_{0}}(V)$. Moreover, if $h$ is chosen as in Lemma 3.1 and if $\mathcal{D}_{s_{0}}^{\prime}$ 
denotes the sheaf of ultra-distributions of order $s_{0}$, we have a commutative diagram

$$
\begin{array}{rrrr}
G^{s, h}(V ; \mathrm{M}) & \hookrightarrow & \mathcal{D}_{s_{0}}^{\prime}(V) \\
\downarrow P(\mathrm{M}) & & & \downarrow P(\mathrm{M}) \\
G^{s, 2^{s} h}(V ; \mathrm{M}) & \hookrightarrow & \mathcal{D}_{s_{0}}^{\prime}(V),
\end{array}
$$

where the horizontal arrows are induced by the injection $\mathcal{C}^{\infty}(V) \hookrightarrow \mathcal{D}_{s_{0}}^{\prime}(V)$ defined by

$$
f \mapsto\left(\psi \mapsto \int_{V} f(x) \psi(x) \mathrm{d} Z(x)\right), f \in \mathcal{C}^{\infty}(V), \psi \in G_{c}^{s_{0}}(V),
$$

and $P(\mathrm{M}): \mathcal{D}_{s_{0}}^{\prime}(V) \longrightarrow \mathcal{D}_{s_{0}}^{\prime}(V)$ is defined by duality. Here we are writing $\mathrm{d} Z \doteq$ $\mathrm{d} Z_{1} \wedge \ldots \wedge \mathrm{d} Z_{N} 1$

We shall now embark upon the proof of two basic results for this work. We recall that we have fixed a Gevrey hypo-analytic structure $\mathfrak{T}$ of order $s_{0}>1$ over $\Omega$. Let $(U, Z) \in \mathfrak{T}$ and let $p \in U$. Composing with a translation allows us to assume that $Z(p)=0$. Next, replacing $Z$ by $A \circ Z$, where $A$ is a non-singular $N \times N$ complex matrix, allows us also to assume that $D_{p} Z$ is the identity. Hence we can contract $U$ around $p$ in order to ensure that $U$ is the domain of a coordinate system $\left(x_{1}, \ldots, x_{N}\right)$ centered at $p$ such that we can write

$$
Z(x)=x+i \Phi(x)
$$

where $\Phi: U \rightarrow \mathbb{R}^{N}$ is a Gevrey map of order $s_{0}$ and satisfies

$$
\Phi(0)=0, \quad D_{0} \Phi=0 .
$$

Contracting further $U$ around $p(=0)$ we can assume that

$$
|\Phi(x)-\Phi(y)| \leq \frac{1}{2}|x-y|, \quad x, y \in U .
$$

A consequence of property (6) is that we can consider the approximation scheme given by the family of entire functions

$$
\mathcal{E}_{\varepsilon}[\psi](z)=\frac{1}{(2 \pi)^{N}} \int_{\mathbb{R}^{N}} \int_{U} \mathrm{e}^{i(z-Z(y)) \cdot \xi-\varepsilon|\xi|^{2}} \psi(y) \mathrm{d} Z(y) \mathrm{d} \xi,
$$

where $\psi \in \mathcal{C}_{c}^{\infty}(U)$.

We have

$$
\mathcal{E}_{\varepsilon}[\psi] \circ Z \stackrel{\varepsilon \rightarrow 0^{+}}{\longrightarrow} \psi \quad \text { in } \mathcal{C}^{\infty}(U) .
$$

Next we introduce a class of "elliptic" ultradifferential operators of class $\left(p !^{s}\right)$.

Definition 3.1. Let $P(\zeta)$ define an ultradifferential operator of class $\left(p !^{s}\right)$. We shall say that $P(\zeta)$ satisfies property (e) if there are constants $\sigma>0, c>0$ such that

$$
|P(\zeta)| \geq c \exp \left\{c|\zeta|^{1 / s}\right\}, \quad \zeta \in \Gamma_{\sigma},
$$

where we have written $\Gamma_{\sigma} \doteq\left\{\zeta=\xi+i \eta: \xi, \eta \in \mathbb{R}^{N},|\eta| \leq \sigma|\xi|\right\}$.

\footnotetext{
${ }^{1}$ Note the integration by parts formulae

$$
\int_{V}\left(\mathrm{M}_{j} \varphi\right)(x) \chi(x) \mathrm{d} Z(x)=-\int_{V} \varphi(x)\left(\mathrm{M}_{j} \chi\right)(x) \mathrm{d} Z(x), \quad j=1, \ldots, N,
$$

which hold for any pair of functions $\varphi, \chi \in \mathcal{C}^{1}(V)$, one of them with compact support (cf. [T2, p.
} 79]). 
(9) is easily seen to be equivalent to the existence of a constant $\gamma>0$ such that

$$
|P(\zeta)| \geq \frac{\gamma^{p+1}|\zeta|^{p}}{p !^{s}}, p \in \mathbb{Z}_{+}, \zeta \in \Gamma_{\sigma} .
$$

We are now in a position to state the two main tools referred to above. In their statements we tacitly assume that we are working in the hypo-analytic chart $(U, Z)$, where $Z$ is given by (4) and satisfies (5) and (6).

Proposition 3.1. Let $P(i \zeta)$ define an ultradifferential operator of class $\left(p !^{s}\right)$, where $s>s_{0}$, satisfying property (e). Given an open neighborhood $V \subset U$ of the origin, there exist $h>0$ and another open neighborhood $W \Subset V$ of the origin such that for every $f \in \mathcal{C}^{\infty}(V)$ there is $u \in G^{s, h}(\bar{W} ; \mathrm{M})$ solving

$$
P(\mathrm{M}) u=f \text { in } W \text {. }
$$

Proof. We first select an open neighborhood $V_{1} \subset V$ of the origin such that

$$
|\Phi(x)-\Phi(y)| \leq \frac{\sigma}{2}|x-y|, \quad x, y \in V_{1},
$$

where $\sigma$ is given as in the definition of property $(\mathfrak{e})$ and assumed $<1$. This is possible thanks again to (5).

Let $f \in \mathcal{C}^{\infty}(V)$ and take $\psi \in \mathcal{C}_{c}^{\infty}\left(V_{1}\right), \psi=1$ in an open neighborhood $W$ of the origin. We assume that $W$ has a smooth boundary. Thanks to the fact that $P(i \zeta)$ satisfies property $(\mathfrak{e})$ we can define the family of entire functions

$$
u_{\varepsilon}(z)=\frac{1}{(2 \pi)^{N}} \int_{\mathbb{R}^{N}} \int_{U} \frac{1}{P(i \xi)} \mathrm{e}^{i(z-Z(y)) \cdot \xi-\varepsilon|\xi|^{2}} \psi(y) f(y) \mathrm{d} Z(y) \mathrm{d} \xi
$$

It is easily seen that

$$
P(\mathrm{M})\left(u_{\varepsilon} \circ Z\right)=\mathcal{E}_{\varepsilon}[\psi f] \circ Z
$$

and consequently (8) gives

$$
P(\mathrm{M})\left[u_{\varepsilon} \circ Z\right] \stackrel{\varepsilon \rightarrow 0^{+}}{\longrightarrow} \psi f
$$

in $\mathcal{C}^{\infty}(V)$

Next we must estimate $u_{\varepsilon}(Z(x))$. For this we first perform an integration by parts in the $y$-integration. For any $q \in \mathbb{Z}_{+}$and any $\alpha \in \mathbb{Z}_{+}^{N}$ and $x \in \bar{W}$ we have

$$
\begin{gathered}
{\left[\mathrm{M}^{\alpha}\left(u_{\varepsilon} \circ Z\right)\right](x)} \\
=\frac{1}{(2 \pi)^{N}} \int_{\mathbb{R}^{N}} \int_{U} \frac{(i \xi)^{\alpha}}{P(i \xi)} \mathrm{e}^{i(Z(x)-Z(y)) \cdot \xi-\varepsilon|\xi|^{2}} \frac{\left(1-\Delta_{\mathrm{M}}\right)^{q}(\psi f)(y)}{\left(1+|\xi|^{2}\right)^{q}} \mathrm{~d} Z(y) \mathrm{d} \xi,
\end{gathered}
$$

where we have written $\Delta_{\mathrm{M}}=\mathrm{M}_{1}^{2}+\ldots+\mathrm{M}_{N}^{2}$.

Next we change the $\xi$-integral to an integration over the chain

$$
\xi \mapsto \zeta \doteq \xi+i \frac{3 \sigma}{4} \frac{x-y}{|x-y|}|\xi| .
$$

Denote by $\mathcal{Q}(x, y, \xi)$ the real part of the exponent after the change $\xi \mapsto \zeta$. Writing

$$
\langle\zeta\rangle^{2}=\zeta_{1}^{2}+\ldots+\zeta_{N}^{2}
$$


we obtain, thanks to (11),

$$
\begin{aligned}
\mathcal{Q}(x, y, \xi) & =-\frac{3 \sigma}{4}|x-y||\xi|-(\Phi(x)-\Phi(y)) \cdot \xi-\varepsilon \operatorname{Re}\langle\zeta\rangle^{2} \\
& \leq-\frac{3 \sigma}{4}|x-y||\xi|-(\Phi(x)-\Phi(y)) \cdot \xi-\varepsilon \frac{7 \sigma^{2}}{16}|\xi|^{2} \\
& \leq-\frac{3 \sigma}{4}|x-y||\xi|+\frac{\sigma}{2}|x-y||\xi| \\
& \leq 0 .
\end{aligned}
$$

Since moreover

$$
\left|1+\langle\zeta\rangle^{2}\right| \geq 1+\frac{7 \sigma^{2}}{16}|\xi|^{2}, \quad|\xi| \leq|\zeta| \leq\left(1+\frac{3 \sigma}{4}\right)|\xi|
$$

and since also the Jacobian of the transformation $\xi \mapsto \zeta$ is homogeneous of degree 0 in $\xi$, (15) and estimates (10) give, for $x \in \bar{W}$,

$$
\left|\left[\mathrm{M}^{\alpha}\left(u_{\varepsilon} \circ Z\right)\right](x)\right| \leq C^{|\alpha|+1}|\alpha| !^{s} \int_{\mathbb{R}^{N}} \int_{U} \frac{\left|\left(1-\Delta_{\mathrm{M}}\right)^{q}(\psi f)(y)\right|}{\left(1+|\xi|^{2}\right)^{q}} \mathrm{~d} y \mathrm{~d} \xi,
$$

where $C>0$ is a suitable constant depending only on $\gamma$ and $\sigma$ but not on $\varepsilon$. Choosing $q>N / 2$ we conclude that $u_{\varepsilon} \circ Z$ is uniformly bounded in $G^{s, C}(\bar{W} ; \mathrm{M})$ and consequently, by Proposition 2.1, given $h>C$ there is a sequence $\varepsilon_{j} \rightarrow 0$ such that $u_{\varepsilon_{j}} \circ Z \rightarrow u$ in $G^{s, h}(\bar{W} ; \mathrm{M})$.

Since finally both convergences $u_{\varepsilon_{j}} \circ Z \rightarrow u, P(\mathrm{M})\left[\left(u_{\varepsilon_{j}}\right) \circ Z\right] \longrightarrow f$ take place in $\mathcal{D}_{s_{0}}^{\prime}(W)$ we conclude that $P(\mathrm{M}) u=f$ in $\mathcal{D}^{\prime}{ }_{s_{0}}(W)$, which concludes the proof.

Remark 3.2. The same conclusion of Proposition 3.1 holds if we only assume that $f \in \mathcal{D}^{\prime}(V)$. Indeed, first we observe that the preceding proof holds if we only assume $f \in \mathcal{C}^{\alpha}(V)$, with $\alpha>N$. Now if $u \in G^{s, h}(\bar{W}, \mathrm{M})$ solves $P(\mathrm{M}) u=f$, then $P(\mathrm{M}) \Delta_{\mathrm{M}}^{p} u=\Delta_{\mathrm{M}}^{p} f$ for any $p \in \mathbb{Z}_{+}$and moreover $\Delta_{\mathrm{M}}^{p} u \in G^{s, h_{p}}(\bar{W}, \mathrm{M})$ for some $h_{p}>0$. Our claim then follows from a local structure theorem proved in [T2, p. 102].

Next we state and prove a regularity result:

Proposition 3.2. Let $P(i \zeta)$ define an ultradifferential operator of class $\left(p !^{s}\right)$, with $s>s_{0}$, satisfying property $(\mathfrak{e})$. Given an open neighborhood $V \subset U$ there is an open neighborhood $D$ of the origin in $\mathbb{C}^{N}$, with $Z^{-1}(D) \Subset V$, such that the following holds:

- For every $v \in \mathcal{C}^{\infty}(V)$ satisfying $P(\mathrm{M}) v=0$ there is $G \in \mathcal{O}(D)$ such that $v=G \circ Z$ in $Z^{-1}(D)$.

In particular any solution $v \in \mathcal{C}^{\infty}(V)$ of the equation $P(\mathrm{M}) v=0$ is $\mathfrak{T}$-hypoanalytic in a fixed neighborhood of the origin.

Proof. We start by fixing a neighborhood $V_{1}$ as at the beginning of the proof of Proposition 3.1 and let $v \in \mathcal{C}^{\infty}(V)$ satisfy $P(\mathrm{M}) v=0$. We have

$$
\int_{V_{1}} v(y)[P(-\mathrm{M}) \psi](y) \mathrm{d} Z(y)=0, \quad \psi \in G_{c}^{s_{0}}\left(V_{1}\right) .
$$


Let $V_{2} \Subset V_{1}$ be another neighborhood of the origin and let $\varphi \in G_{c}^{s_{0}}\left(V_{1}\right)$ be identically equal to one in $V_{2}$. If $H \in \mathcal{O}\left(\mathbb{C}^{N}\right)$, then

$$
\begin{gathered}
P(-\mathrm{M})[\varphi(H \circ Z)] \\
=\varphi P(-\mathrm{M})(H \circ Z)+\sum_{\alpha \geq 0}(-1)^{|\alpha|} a_{\alpha} \sum_{\beta \leq \alpha,|\beta|>0}\left(\begin{array}{c}
\alpha \\
\beta
\end{array}\right) \mathrm{M}^{\beta} \varphi \mathrm{M}^{\alpha-\beta}(H \circ Z) .
\end{gathered}
$$

Now let $\delta>0$ be a small number and define $K_{\delta}$ as the set of all vectors in $\mathbb{C}^{N}$ whose Euclidean distance to $Z\left(\overline{V_{1}} \backslash V_{2}\right)$ is $\leq \delta$. Noticing that

$$
\mathrm{M}^{\alpha-\beta}(H \circ Z)=\left(\partial_{z}^{\alpha-\beta} H\right) \circ Z
$$

Cauchy estimates give

$$
\sup _{\overline{V_{1}} \backslash V_{2}}\left|\mathrm{M}^{\alpha-\beta}(H \circ Z)\right| \leq \frac{|\alpha-\beta| !}{\delta^{|\alpha-\beta|}} \sup _{K_{\delta}}|H| .
$$

Hence (19) in conjunction with (2) gives, for some constant $C>0$,

$$
\begin{gathered}
\left|\int_{V_{1}} v(y)\{P(-\mathrm{M})[\varphi(H \circ Z)]-\varphi P(-\mathrm{M})(H \circ Z)\}(y) \mathrm{d} Z(y)\right| \\
\leq \sup _{K_{\delta}}|H| \sum_{\alpha \geq 0} \frac{C^{|\alpha|+1}}{\delta|\alpha||\alpha| !^{s-s_{0}}} \sum_{\beta \leq \alpha,|\beta|>0}\left(\begin{array}{c}
\alpha \\
\beta
\end{array}\right) \frac{|\beta| !^{s_{0}}|\alpha-\beta| !}{|\alpha| !^{s_{0}}} \\
\leq \sup _{K_{\delta}}|H| \sum_{\alpha \geq 0} \frac{2^{|\alpha|} C^{|\alpha|+1}}{\delta^{|\alpha|}|\alpha| !^{s-s_{0}}} .
\end{gathered}
$$

Hence we have reached the following conclusion: if $v \in \mathcal{C}^{\infty}(V)$ satisfies $P(\mathrm{M}) v=$ 0 and if $V_{2}, V_{1}$ and $\varphi$ are chosen as in the preceding discussion, then for every $\delta>0$ small there is a constant $C_{\delta}>0$ such that

$$
\left|\int_{V_{1}} v(y) \varphi(y)[P(-\mathrm{M})(H \circ Z)](y) \mathrm{d} Z(y)\right| \leq C_{\delta} \sup _{K_{\delta}}|H| .
$$

We then apply (20) with $H$ replaced by

$$
w \mapsto H_{\varepsilon}(z, w) \doteq \frac{1}{(2 \pi)^{N}} \int_{\mathbb{R}^{N}} \frac{1}{P(-i \xi)} \mathrm{e}^{i(z-w) \cdot \xi-\varepsilon|\xi|^{2}} \mathrm{~d} \xi,
$$

with $z \in \mathbb{C}^{N}$ regarded as a parameter and $\varepsilon>0$. It is clear that

$$
P(-\mathrm{M})\left[H_{\varepsilon}(z, Z(y)]=\frac{1}{(2 \pi)^{N}} \int_{\mathbb{R}^{N}} \mathrm{e}^{i(z-Z(y)) \cdot \xi-\varepsilon|\xi|^{2}} \mathrm{~d} \xi\right.
$$

and then we obtain

$$
\left|\mathcal{E}_{\varepsilon}[\varphi v](z)\right| \leq C_{\delta} \sup _{w \in K_{\delta}}\left|H_{\varepsilon}(z, w)\right|
$$

with $C_{\delta}$ independent of both $z$ and $\varepsilon$. In order to conclude the proof of the proposition we must show that there are $D$, an open neighborhood of the origin in $\mathbb{C}^{N}$, with $Z^{-1}(D) \subset V_{2}$, and $\delta>0$ small such that

$$
\sup _{w \in K_{\delta}}\left|H_{\varepsilon}(z, w)\right| \leq \text { const., } \quad z \in D,
$$


uniformly in $\varepsilon>0$. Indeed if this is so, (21) would imply the existence of a sequence $\varepsilon_{j} \rightarrow 0$ and of a function $G \in \mathcal{O}(D)$ such that

$$
\mathcal{E}_{\varepsilon_{j}}[\varphi v](z) \longrightarrow G \text { in } \mathcal{O}(D) .
$$

From (8) we have $v=G \circ Z$ in $Z^{-1}(D)$, whence our claim.

We are then left with the proof of (22). We write $w \in K_{\delta}$ in the form $w=$ $Z(y)+w^{\prime}, y \in \bar{V}_{1} \backslash V_{2},\left|w^{\prime}\right| \leq \delta$ and also $z$ in the form $z=Z(x)+i \gamma$, with $\gamma \in \mathbb{R}^{N}$. As in the proof of Proposition 3.1, in the integral that defines $H_{\varepsilon}$ we perform the change of variables (16). Denoting now by $\mathcal{Q}_{1}(z, w, \xi)$ the real part of the exponent obtained after the change $\xi \mapsto \zeta$ we obtain, as before,

$$
\mathcal{Q}_{1}(z, w, \xi) \leq-\frac{\sigma}{2}|x-y||\xi|+\mathrm{O}\left(\left(|\gamma|+\left|w^{\prime}\right|\right)|\xi|\right) .
$$

It is then clear that if $D$ and $\delta>0$ are chosen appropriately small we obtain, for some constant $c>0$,

$$
\mathcal{Q}_{1}(z, w, \xi) \leq-c|\xi|
$$

when $z \in D, w \in K_{\delta}, \xi \in \mathbb{R}^{N}$ and then (22) follows from the fact that $P(-i \zeta)$ has a strictly positive lower bound in $\Gamma_{\sigma}$.

The proof of Proposition 3.2 is now complete.

Remark 3.3. It is easily seen that the conclusion of Proposition 3.2 remains true if we assume that $v \in \mathcal{D}^{\prime}(V)$.

Remark 3.4. The conclusion of Proposition 3.1 remains true even if we assume that $v \in \mathcal{D}_{s_{0}}^{\prime}(V)$. In this case it is necessary to extend the argument a little further (in particular we must know that the approximation in (8) occurs in $G^{s}$ if $\psi$ is Gevrey of order $s>1$, a result proved in $\mathrm{Co}$ ). The details are left to the interested reader.

We conclude this section with an example. Consider the following entire function:

$$
\mathrm{Q}_{s}(\zeta)=\prod_{p=1}^{\infty}\left(1-\frac{1}{p^{2 s}}\left[\zeta_{1}^{2}+\ldots+\zeta_{N}^{2}\right]\right) .
$$

It is proved in $\left[\mathrm{Ko}\right.$ that $Q_{s}(i \zeta)$ defines indeed an ultradifferential operator of class $\left(p !^{s}\right)$. Moreover we can prove

Lemma 3.3. $\mathrm{Q}_{s}(i \zeta)$ satisfies property $(\mathfrak{e})$. More precisely, given $\left.\sigma \in\right] 0,1[$ and setting $\sigma_{0}=\left\{\left(1-\sigma^{2}\right) /\left(1+\sigma^{2}\right)\right\}^{1 / 2}$, then

$$
\left|\mathrm{Q}_{s}(i \zeta)\right| \geq \frac{|\zeta|^{p} \sigma_{0}^{p}}{p !^{s}}, \quad p \in \mathbb{Z}_{+}, \zeta \in \Gamma_{\sigma} .
$$

Proof. First we observe that if $\zeta=\xi+i \eta \in \Gamma_{\sigma}$, with $\xi, \eta$ real, then

$$
\operatorname{Re}\langle\zeta\rangle^{2}=|\xi|^{2}-|\eta|^{2} \geq\left(1-\sigma^{2}\right)|\xi|^{2} \geq \sigma_{0}^{2}|\zeta|^{2} .
$$

In particular we obtain

$$
\left|1+\frac{1}{q^{2 s}}\langle\zeta\rangle^{2}\right| \geq 1+\frac{1}{q^{2 s}} \operatorname{Re}\langle\zeta\rangle^{2} \geq 1+\frac{\sigma_{0}^{2}}{q^{2 s}}|\zeta|^{2} \geq 1
$$

and then, for every $p \in \mathbb{Z}_{+}$,

$$
\left|Q_{s}(i \zeta)\right| \geq \prod_{q=1}^{p}\left(1+\frac{\sigma_{0}^{2}}{q^{2 s}}|\zeta|^{2}\right) \geq \prod_{q=1}^{p} \frac{\sigma_{0}|\zeta|}{q^{s}}=|\zeta|^{p} \sigma_{0}^{p} p !^{-s} .
$$




\section{Gevrey locally integrable structures}

Let $s \geq 1$. Given an open subset $\Omega$ of $\mathbb{R}^{N}$, a Gevrey locally integrable structure of order $s$ is a subbundle $\mathrm{T}^{\prime}$ of $\mathbb{C} \mathrm{T}^{*} \Omega$ of rank $m$ which is locally generated by the differentials of $m$ Gevrey functions of order $s$. Here we are writing $\mathbb{C T}^{*} \Omega$ to denote the complexified cotangent bundle over $\Omega$.

To every such structure there is canonically associated a complex of differential operators whose study of the Gevrey local solvability is our main goal.

The invariant construction of this complex can be found in [T2, Section I.6] (see also $[\mathrm{BCH}$, chapter 7$]$ ). Here we recall its standard local representation in a suitable choice of coordinates.

We assume that the origin belongs to $\Omega$ and write $N=m+n$. According to T2, p. 43] we can find a local system of coordinates $(x, t)=\left(x_{1}, \ldots, x_{m}, t_{1}, \ldots, t_{n}\right)$ centered at the origin and defined in a domain of the form $U=B \times \Theta$, where $B$ (resp. $\Theta$ ) is an open ball centered at the origin in $\mathbb{R}^{m}$ (resp. $\mathbb{R}^{n}$ ), as well as a $G^{s}$ mapping $\phi: \Omega \rightarrow \mathbb{R}^{m}, \phi=\left(\phi_{1}, \ldots, \phi_{m}\right)$ such that if we set

$$
Z_{k}(x, t)=x_{k}+i \phi_{k}(x, t), \quad k=1, \ldots, m,
$$

then

$$
Z(0,0)=0, Z_{x}(0,0)=\text { identity, } \operatorname{det} Z_{x} \neq 0 \text { in } U
$$

and

$$
\left\{\mathrm{d} Z_{1}, \ldots, \mathrm{d} Z_{m}\right\} \text { span } \mathrm{T}^{\prime} \text { over } U \text {. }
$$

We shall set $Z(x, t)=\left(Z_{1}(x, t), \ldots, Z_{m}(x, t)\right)$.

Next we introduce the complex vector fields $\mathrm{X}_{1}, \ldots, \mathrm{X}_{m}$ on $U$ defined by the relations

$$
\mathrm{X}_{k} Z_{\ell}=\delta_{k \ell}, \mathrm{X}_{k} t_{j}=0, k, \ell=1, \ldots, m, j=1, \ldots, n
$$

and define

$$
\mathrm{L}_{j}=\frac{\partial}{\partial t_{j}}-i \sum_{k=1}^{m} \frac{\partial \phi_{k}}{\partial t_{j}}(x, t) \mathrm{X}_{k}, \quad j=1, \ldots, n .
$$

Since $\mathrm{L}_{j} Z_{k}=0, j=1, \ldots, n, k=1, \ldots, m$ it follows that the orthogonal of $\mathrm{T}^{\prime}$, which is a subbundle of $\mathbb{C T} \Omega$ of rank $n$, is spanned over $U$ by the vector fields $\mathrm{L}_{j}$. Notice also that the vector fields $\mathrm{X}_{1}, \ldots, \mathrm{X}_{m}, \mathrm{~L}_{1}, \ldots, \mathrm{L}_{n}$ are linearly independent and pairwise commuting.

Given $V \subset U$ open and $p \in\{0,1, \ldots, n\}$ we introduce the spaces

$$
\mathcal{C}^{\infty}\left(V ; \underline{\Lambda}^{p}\right) \doteq\left\{u=\sum_{|J|=p} u_{J}(x, t) \mathrm{d} t_{J}, u_{J} \in \mathcal{C}^{\infty}(V)\right\}
$$

where the standard notation for differential forms is employed.

We then define differential operators

$$
\mathbb{L}: \mathcal{C}^{\infty}\left(V, \underline{\Lambda}^{p}\right) \longrightarrow \mathcal{C}^{\infty}\left(V, \underline{\Lambda}^{p+1}\right)
$$

by the expression

$$
\mathbb{L}(u)=\sum_{|J|=p} \sum_{j=1}^{n}\left(\mathrm{~L}_{j} u_{J}\right) \mathrm{d} t_{j} \wedge \mathrm{d} t_{J}
$$


Notice that $\left[L_{j}, L_{k}\right]=0$ for all $j$ and $k$ implies that $\mathbb{L}^{2}=0$. Hence (25) defines a complex of differential operators, which is referred to as the differential complex associated to the locally integrable structure $\mathrm{T}^{\prime}$.

We can also introduce the spaces $G^{s}\left(V, \underline{\Lambda}^{p}\right)$ of all forms of the kind

$$
u=\sum_{|J|=p} u_{J}(x, t) \mathrm{d} t_{J},
$$

where now $u_{J} \in G^{s}(V)$ Since the coefficients of the vector fields $\mathrm{L}_{j}$ are $G^{s}$ we obtain a new complex of differential operators

$$
\mathbb{L}: G^{s}\left(V, \underline{\Lambda}^{p}\right) \longrightarrow G^{s}\left(V, \underline{\Lambda}^{p+1}\right) .
$$

\section{Gevrey solvability}

We return to the differential complex (25) and recall the following standard definition. Fix $q \in\{1, \ldots, n\}$.

Definition 5.1. We say that the system $\mathbb{L}$ is smoothly solvable at the origin in degree $q$ if for every open neighborhood $U_{0} \subset U$ of the origin there is another such neighborhood $V_{0} \subset U_{0}$ such that the following is true: for every $f \in \mathcal{C}^{\infty}\left(U_{0} ; \underline{\Lambda}^{q}\right)$ satisfying $\mathbb{L} f=0$ there is $u \in \mathcal{C}^{\infty}\left(V_{0} ; \underline{\Lambda}^{q-1}\right)$ satisfying $\mathbb{L} u=f$ in $V_{0}$.

Remark 5.1. Smooth solvability follows from a much weaker solvability concept. Indeed if for every open neighborhood $U_{0} \subset U$ of the origin and for every $f \in$ $\mathcal{C}^{\infty}\left(U_{0} ; \underline{\Lambda}^{q}\right)$ satisfying $\mathbb{L} f=0$ there is $u \in \mathcal{D}^{\prime}\left(U_{0} ; \underline{\Lambda}^{q-1}\right)$ solving $\mathbb{L} u=f$ in some neighborhood of the origin (which can depend on $f$ ), then $\mathbb{L}$ is smoothly solvable at the origin in degree $q$. The proof follows from a category argument and from [T2, p. 404].

This section will be devoted to the proof of the following theorem:

Theorem 5.1. Assume that $\mathrm{T}^{\prime}$ defines a Gevrey locally integrable structure of order $s_{0}>1$ and let $s>s_{0}$. If $\mathbb{L}$ is smoothly solvable at the origin in degree $q$, for some $q \in\{1, \ldots, n\}$, then for every open neighborhood $U_{1} \subset U$ there is another such neighborhood $V_{1} \subset U_{1}$ such that the following is true: for every $f \in G^{s}\left(U_{1} ; \underline{\Lambda}^{q}\right)$ satisfying $\mathbb{L} f=0$ there is $u \in G^{s}\left(V_{1} ; \underline{\Lambda}^{q-1}\right)$ satisfying $\mathbb{L} u=f$ in $V_{1}$.

Before we embark upon the proof we make some remarks. First we observe that property (23) allows us to consider the Gevrey hypo-analytic structure $\mathfrak{T}$ of class $s_{0}$ on $U$ defined by the hypo-analytic chart $(U, \lambda)$, where $\lambda: U \rightarrow \mathbb{C}^{N}$ is defined by $\lambda(x, t)=(Z(x, t), t)$. Although we cannot assert that $D(\operatorname{Im} \lambda)$ vanishes at the origin, this drawback can be easily overcome. We consider the map $\lambda_{\sharp}: U \rightarrow \mathbb{C}^{N}$ defined by

$$
\lambda_{\sharp}(x, t)=\left(Z(x, t)-i \phi_{t}(0) t, t\right) .
$$

Notice that $\lambda_{\sharp}=A \circ \lambda$, with $A$ a non-singular $N \times N$ complex matrix, which gives $\left(U, \lambda_{\sharp}\right) \in \mathfrak{T}$. Notice also that now we have $\lambda_{\sharp}(0,0)=0$ and $D\left(\operatorname{Im} \lambda_{\sharp}\right)=0$ at the origin. The corresponding operators for the hypo-analytic chart $\left(U, \lambda_{\sharp}\right)$ are given by

$$
\mathrm{X}_{1}, \ldots, \mathrm{X}_{m}, \mathrm{~L}_{1}^{\sharp}, \ldots, \mathrm{L}_{n}^{\sharp},
$$

\footnotetext{
${ }^{2}$ More generally, if $E(V)$ is a subspace of $\mathcal{D}^{\prime}(V)$ we denote by $E\left(V ; \underline{\Lambda}^{q}\right)$ the space of all currents of the form (27), where $u_{J} \in E(V)$ for all $J$.
} 
where

$$
\mathrm{L}_{j}^{\sharp}=\mathrm{L}_{j}+i \sum_{k=1}^{m} \frac{\partial \phi_{k}}{\partial t_{j}}(0,0) \mathrm{X}_{k} .
$$

This discussion, together with Lemma 3.3, shows that we can take $U$ sufficiently contracted around the origin in order that the conclusions of both Propositions 3.1 and 3.2 hold for the ultradifferential operator of class $\left(p !^{s}\right)\left(s>s_{0}\right)$ defined by

$$
\mathrm{Q}_{s, r}\left(\mathrm{X}, \mathrm{L}^{\sharp}\right)=\prod_{p=1}^{\infty}\left(1-\frac{r^{2}}{p^{2 s}}\left[\mathrm{X}_{1}^{2}+\ldots+\mathrm{X}_{m}^{2}+\left(\mathrm{L}_{1}^{\sharp}\right)^{2}+\ldots+\left(\mathrm{L}_{n}^{\sharp}\right)^{2}\right]\right) .
$$

The key property of such operators is

$$
\mathrm{Q}_{s, r}\left(\mathrm{X}, \mathrm{L}^{\sharp}\right) \mathrm{L}_{j}=\mathrm{L}_{j} \mathrm{Q}_{s, r}\left(\mathrm{X}, \mathrm{L}^{\sharp}\right) \quad \text { in } \mathcal{D}_{s_{0}}^{\prime}(U)
$$

for all $j=1, \ldots, n$, a consequence of the fact that each $\mathrm{L}_{j}$ commutes with all $\mathrm{X}_{k}$, $\mathrm{L}_{\ell}^{\sharp}$.

Proof of Theorem 5.1. In what follows we shall make the operator $\mathrm{Q}_{s, r}\left(\mathrm{X}, \mathrm{L}^{\sharp}\right)$ to act on forms by acting componentwise. Take $U_{1}$ and $f \in G^{s}\left(U_{1} ; \underline{\Lambda}^{q}\right)$ as in the statement and select an open neighborhood of the origin $U_{0} \Subset U_{1}$. By Lemma 3.1 and Remark 3.1, it follows that there is $r>0$ such that $\mathrm{Q}_{s, r}\left(\mathrm{X}, \mathrm{L}^{\sharp}\right) f \in \mathcal{C}^{\infty}\left(\overline{U_{0}} ; \underline{\Lambda}^{q}\right)$. By hypothesis there exist an open neighborhood of the origin $V_{0} \subset U_{0}$ (which depends only on $U_{0}$ and not on $\left.f\right)$ and $w \in \mathcal{C}^{\infty}\left(\overline{V_{0}} ; \underline{\Lambda}^{q-1}\right)$ such that $\mathbb{L} w=\mathrm{Q}_{s, r}\left(\mathrm{X}, \mathrm{L}^{\sharp}\right) f$ in $V_{0}$ (notice that $\mathbb{L} Q_{s, r}\left(\mathrm{X}, \mathrm{L}^{\sharp}\right) f=0$ thanks to (29)). Next we apply Proposition 3.1: there exist an open neighborhood of the origin $W \subset V_{0}$ (depending only on $V_{0}$ and $r), h_{r}>0$ and $v \in \mathcal{C}^{\infty}\left(W ; \underline{\Lambda}^{q-1}\right)$, with coefficients in $G^{s, h_{r}}\left(\bar{W} ; \mathrm{X}, \mathrm{L}^{\sharp}\right)$, such that $\mathrm{Q}_{s, r}\left(\mathrm{X}, \mathrm{L}^{\sharp}\right) v=w$ in $W$. As before it follows that

$$
\begin{aligned}
\mathrm{Q}_{s, r}\left(\mathrm{X}, \mathrm{L}^{\sharp}\right)[\mathbb{L} v-f] & =\mathbb{L} \mathrm{Q}_{s, r}\left(\mathrm{X}, \mathrm{L}^{\sharp}\right) v-\mathrm{Q}_{s, r}\left(\mathrm{X}, \mathrm{L}^{\sharp}\right) f \\
& =\mathbb{L} w-\mathrm{Q}_{s, r}\left(\mathrm{X}, \mathrm{L}^{\sharp}\right) f \\
& =0
\end{aligned}
$$

in $W$. Write

$$
g=\sum_{|J|=q} g_{J}(x, t) \mathrm{d} t_{J} \doteq \mathbb{L} v-f
$$

and apply Proposition 3.2, recalling that $\lambda_{\sharp}=A \circ \lambda$, with $A$ an $N \times N$ complex matrix: there is an open polydisc $\Delta$ centered at the origin in $\mathbb{C}^{N}$, with $\lambda^{-1}(\Delta) \subset W$ and, for each $J$ a function $G_{J} \in \mathcal{O}(\Delta)$ such that $g_{J}=G_{J} \circ \lambda$. Hence $g=\lambda^{*}(G)$, where

$$
G(z, \zeta) \doteq \sum_{|J|=p} F_{J}(z, \zeta) \mathrm{d} \zeta_{J}
$$

and the coordinates in $\mathbb{C}^{N}$ are written as $(z, \zeta)$, with $z=\left(z_{1}, \ldots, z_{m}\right)$ and $\zeta=$ $\left(\zeta_{1}, \ldots, \zeta_{n}\right)$. Here $\Delta$ depends only on $W$.

Notice that

$$
0=\mathbb{L} g=\mathbb{L} \lambda^{*}(G)=\lambda^{*}\left(\partial_{\zeta} G\right)
$$

and consequently, by a uniqueness argument [T2, p. 90], we conclude that $\partial_{\zeta} G=0$ in $\Delta$. Finally we solve, in $\Delta$, the equation

$$
\partial_{\zeta} H=G
$$


where

$$
H(z, \zeta)=\sum_{|K|=p-1} H_{K}(z, \zeta) \mathrm{d} \zeta_{K}
$$

and the coefficients $H_{K}$ are holomorphic in $\Delta$. Finally we set $h \doteq \lambda^{*}(H)$. It follows that $h \in G^{s_{0}}\left(V_{1} ; \underline{\Lambda}^{p-1}\right)$ and that

$$
\mathbb{L} h=\lambda^{*}\left(\partial_{\zeta} H\right)=\lambda^{*}(G)=\mathbb{L} v-f .
$$

Hence

$$
u \doteq v-h \in G^{s}\left(V_{1} ; \underline{\Lambda}^{p-1}\right)
$$

solves $\mathbb{L} u=f$ in $V_{1}$. The proof is complete.

Remark 5.2. Notice that the restriction $s>s_{0}$ in the statement of Theorem 5.1 is a technical one. It is related to the fact that ultradifferential operators satisfying (2) do not define endomorphisms of $G^{s}(\Omega)$, which has forced us to make all the analyses that follow Lemma 3.2.

Remark 5.3. As kindly pointed out to us by the referee, there is an interesting generalization of Theorem 5.1 which can be proved along exactly the same lines. Denote the coordinates in $\mathbb{C}^{N}$ by $z=\left(z_{1}, \ldots, z_{N}\right)$.

Theorem 5.2. Let $X \subset \mathbb{C}^{N}$ be a maximally real submanifold of class $G^{s_{0}}$ and let $A\left(\partial_{z}\right)$ be a differential system with constant coefficients. If the induced system $A_{X}$ on $X$ is locally smoothly solvable, then it is locally solvable in $G^{s}$ for every $s>s_{0}$.

Theorem 5.1 follows from Theorem 5.2 when we take as $A\left(\partial_{z}\right)$ a partial de Rham system $A\left(\partial_{z}\right)=\left(\partial / \partial z_{1}, \ldots, \partial / \partial z_{p}\right)$.

\section{GeVRey SOLVABILITY FOR FIRST ORDER LINEAR PDE}

Let

$$
P(y, D)=\sum_{j=1}^{N} a_{j}(y) \partial / \partial y_{j}+b(y)
$$

be a first order differential operator defined on an open subset $\Omega$ of $\mathbb{R}^{N}$. We assume that the coefficients of $P(x, D)$ are real-analytic in $\Omega$ and that $P(x, D)$ is of principal type, that is,

$$
\sum_{j=1}^{N}\left|a_{j}(y)\right| \neq 0, \quad \forall y \in \Omega .
$$

We can prove

Theorem 6.1. Let $y_{0} \in \Omega$ and assume that $P(y, D)$ satisfies the Nirenberg-Treves condition $(\mathcal{P})$ at $y_{0}$. Then for every $s>1$ and every $f \in G^{s}$ in a neighborhood of $y_{0}$ there is $u \in G^{s}$ in a neighborhood of $y_{0}$ solving $P(y, D) u=f$.

We refer to $[\mathrm{NT}]$ for the original statement of condition $(\mathcal{P})$ for first order differential operators.

Proof. The proof is a consequence of Theorem 5.1. Let us write

$$
\mathrm{Y}=\sum_{j=1}^{N} a_{j}(y) \partial / \partial y_{j}
$$


The complex vector field $\mathrm{Y}$ has no singularities, and then we can find a real-analytic function $H$, defined in a neighborhood of $y_{0}$, solving $\mathrm{Y} H=b$. But then

$$
P(y, D)=\exp \{-H\} Y \exp \{H\},
$$

which shows that it suffices to prove the result for $\mathrm{Y}$ substituted for $P(y, D)$.

We now invoke the Cauchy-Kovalevsky theorem: writing $N=m+1$, there are real-analytic functions $W_{1}, \ldots, W_{m}$ defined in an open neighborhood of $y_{0}$ such that $\mathrm{Y} W_{k}=0, k=1, \ldots, m$, and $\mathrm{d} W_{1}, \ldots, \mathrm{d} W_{m}$ are $\mathbb{C}$-linearly independent at each point.

Consequently, the vector field $\mathrm{Y}$ spans the orthogonal to the real-analytic locally integrable structure generated by $\mathrm{d} W_{1}, \ldots, \mathrm{d} W_{m}$, and we can recall what was described in Section 3: we can find a local system of cooordinates $(x, t)=$ $\left(x_{1}, \ldots, x_{m}, t\right)$ centered at $y_{0}$ and defined in a domain of the form $\left.U=B \times\right]-\delta, \delta[$, where $B$ is an open ball centered at the origin in $\mathbb{R}^{m}$, as well as a real-analytic function $\phi: \Omega \rightarrow \mathbb{R}^{m} \phi=\left(\phi_{1}, \ldots, \phi_{m}\right)$, satisfying

$$
\phi(0,0)=0, \quad \phi_{x}(0,0)=0,
$$

such that if we set

$$
Z_{k}(x, t)=x_{k}+i \phi_{k}(x, t), \quad k=1, \ldots, m,
$$

then $\left\{\mathrm{d} W_{1}, \ldots, \mathrm{d} W_{k}\right\}$ and $\left\{\mathrm{d} Z_{1}, \ldots, \mathrm{d} Z_{k}\right\}$ span the same subbundle of $\mathbb{C T}^{*} U$. If we again introduce the complex vector fields $\mathrm{X}_{1}, \ldots, \mathrm{X}_{m}$ on $U$ defined by the relations

$$
\mathrm{X}_{k} Z_{\ell}=\delta_{k \ell}, \mathrm{X}_{k} t=0, k, \ell=1, \ldots, m
$$

and define

$$
\mathrm{L}=\frac{\partial}{\partial t}-i \sum_{j=1}^{m} \frac{\partial \phi_{j}}{\partial t}(x, t) \mathrm{X}_{j}
$$

then $\mathrm{Y}$ equals $\mathrm{L}$ up to a non-vanishing real-analytic factor. This reduces the Gevrey solvability of $P(y, D)$ to that of $\mathrm{L}$. But, by the invariance of condition $(\mathcal{P})$, we know that $\mathrm{L}$ also satisfies condition $(\mathcal{P})$ and then $\mathrm{L}$ is smoothly solvable at the origin (cf. [T1, $[\mathrm{H}]$ ). Our conclusion then follows from Theorem 5.1.

Remark 6.1. Another proof of Theorem 6.1 can be devised by studying in detail the (local) right inverse for $P(y, D)$ constructed in [T1]. Our argument though allows us to state a stronger result. If we just assume that the coefficients of $P(y, D)$ are $G^{s_{0}}$ and if we assume, in addition to condition $(\mathcal{P})$, that the vector field $\mathrm{Y}$ is $G^{s_{0}}$-integrable, in the sense that in a neighborhood of $y_{0}$ there are defined functions $W_{1}, \ldots, W_{m}$ of class $G^{s_{0}}$ such that $\mathrm{Y} W_{k}=0, k=1, \ldots, m$, and $\mathrm{d} W_{1}, \ldots, \mathrm{d} W_{m}$ are $\mathbb{C}$-linearly independent at each point, then the conclusion of Theorem 6.1 holds for every $s>s_{0}$.

\section{REPRESENTATION OF SOLUTIONS AND L-CLOSED FORMS}

We keep the hypotheses and notation established in Sections 3, 4 and 5.

Theorem 7.1. Assume that $\mathrm{T}^{\prime}$ defines a Gevrey locally integrable structure of order $s_{0}>1$ and let $s>s_{0}, r>0$. Then for every open neighborhood $V \subset U$ of the origin 
there exist another such neighborhood $W \Subset V$ and an open polydisk $D$ centered at the origin in $\mathbb{C}^{m}$, with $W \subset Z^{-1}(D)$, such that the following is true:

- For every $u \in \mathcal{D}^{\prime}(V)$ such that $\mathbb{L} u=0$ there are $g \in G^{s}(W)$ satisfying $\mathbb{L} g=0$ and $H \in \mathcal{O}(D)$ such that

$$
u=\mathrm{Q}_{s, r}\left(\mathrm{X}, \mathrm{L}^{\sharp}\right) g+H \circ Z \quad \text { in } W .
$$

Proof. The argument is similar to that in the proof of Theorem 5.1, to which we also refer for the notation. First select an open neighborhood $V_{1} \Subset V$ of the origin. By Proposition 3.1 (cf. Remark 3.2) we can write

$$
u=\mathrm{Q}_{s, r}\left(\mathrm{X}, \mathrm{L}^{\sharp}\right) v \text { in } V_{1}
$$

for some $v \in G^{s}\left(V_{1}\right)$. We have

$$
0=\mathbb{L} u=\mathrm{Q}_{s, r} \mathbb{L} v
$$

and then, arguing as in the proof of Theorem 5.1, we can find an open neighborhood $W \subset V_{1}$ of the origin, an open polydisc $\Delta \in \mathbb{C}^{N}$, centered at the origin and with $W \subset \lambda^{-1}(\Delta)$, both depending only on $V_{1}$, and $G \in \mathcal{O}(\Delta)$ such that $\mathbb{L}(G \circ \lambda)=\mathbb{L} v$ in $W$. Then, setting $g \doteq v-G \circ \lambda$, (31) gives

$$
u=\mathrm{Q}_{s, r} g+H \circ \lambda,
$$

in $W$, with $H$ also holomorphic in $\Delta$. But since $\mathbb{L} u=\mathbb{L} g=0$ we obtain $\mathbb{L}(H \circ \lambda)=$ $\lambda^{*}\left(\partial_{\zeta} H\right)=0$. This implies $\partial_{\zeta} H=0$ in $\Delta$ and consequently $H$ depends only on $z_{1}, \ldots, z_{m}$, which gives (30).

Likewise we can prove:

Theorem 7.1'. Assume that $\mathrm{T}^{\prime}$ defines a locally Gevrey locally integrable structure of order $s_{0}>1$ and let $s>s_{0}, r>0$ and $q \in\{1, \ldots, n\}$. Then for every open neighborhood $V \subset U$ of the origin there is another such neighborhood $W \Subset V$ such that the following is true:

- For every $u \in \mathcal{D}^{\prime}\left(V ; \underline{\Lambda}^{q}\right)$ such that $\mathbb{L} u=0$ there exist $g \in G^{s}\left(W ; \underline{\Lambda}^{q}\right)$ satisfying $\mathbb{L} g=0$ and $h \in \mathcal{A}_{\mathfrak{T}}\left(W ; \underline{\Lambda}^{q-1}\right)$ such that

$$
u=\mathrm{Q}_{s, r}\left(\mathrm{X}, \mathrm{L}^{\sharp}\right) g+\mathbb{L} h \quad \text { in } W .
$$

\section{GeVRey REGUlarity}

We once more return to the situation described in Sections 4 and 5. In particular we are assuming that the locally integrable structure $\mathrm{T}^{\prime}$ is Gevrey of order $s$.

Our goal in this section is just to present an example of how the techniques presented in this work can be applied to the study of Gevrey regularity for the solutions of the operator $\mathbb{L}$ acting on (scalar) ultra-distributions (cf. Theorem 8.1 below).

In order to do so we start by recalling the following definition:

Definition 8.1. We shall say that $\mathbb{L}$ is $G^{s}$-hypoelliptic at the origin if for every $V \subset U$ and every $u \in \mathcal{D}_{s}^{\prime}(V)$, the fact that $\mathrm{L}_{j} u$ is $G^{s}$ in a neighborhood of the origin, $j=1, \ldots, n$, implies that $u$ itself is of class $G^{s}$ in such a neighborhood.

When $s=1$ we must interpret $\mathcal{D}_{1}^{\prime}(V)$ as the space of hyperfunctions in $V$. In this case we obtain a stronger concept of analytic-hypoellipticity at the origin than that introduced in [T2, p. 146]. 
Next we introduce a concept of (weak)-regularity.

Definition 8.2. We shall say that $\mathbb{L}$ satisfies property $\mathfrak{h}_{s}$ at the origin if for every $V \subset U$ and every $u \in \mathcal{D}_{s}^{\prime}(V)$ the fact that $\mathrm{L}_{j} u$ is smooth in a neighborhood of the origin, $j=1, \ldots, n$, implies that $u$ itself is a (Schwartz) distribution in a neighborhood of the origin.

Using arguments similar to the ones used in the proof of Theorem 4.1, to which we refer in the following proof, we conclude this work by proving:

Theorem 8.1. If $\mathrm{T}^{\prime}$ is Gevrey of order $s_{0}>1$ and if $\mathbb{L}$ satisfies property $\mathfrak{h}_{s_{0}}$ at the origin, then, for every $s>s_{0}, \mathbb{L}$ is $G^{s}$-hypoelliptic at the origin.

Proof. Let $V \subset U$ be an open neighborhood of the origin and let $u \in \mathcal{D}_{s}^{\prime}(V)$ be such that $f \doteq \mathbb{L} u$ is Gevrey of order $s$ in a neighborhood of the origin. Select $V_{1} \Subset V$, a neighborhood of the origin, and $h>0$ so that $\left.f\right|_{V_{1}}$ has coefficients in $G^{s, h}\left(\overline{V_{1}} ; \mathrm{X}, \mathrm{L}^{\sharp}\right)$. Also select $r>0$ such that

$$
\mathrm{Q}_{s, r}\left(\mathrm{X}, \mathrm{L}^{\sharp}\right) f \in \mathcal{C}^{\infty}\left(\overline{V_{1}}, \underline{\Lambda}^{1}\right) .
$$

We have, in $\mathcal{D}_{s_{0}}^{\prime}\left(V_{1}\right)$,

$$
\mathrm{Q}_{s, r}\left(\mathrm{X}, \mathrm{L}^{\sharp}\right) f=\mathrm{Q}_{s, r}\left(\mathrm{X}, \mathrm{L}^{\sharp}\right) \mathbb{L} u=\mathbb{L} \mathrm{Q}_{s, r}\left(\mathrm{X}, \mathrm{L}^{\sharp}\right) u .
$$

Since $\mathbb{L}$ satisfies property $\mathfrak{h}_{s_{0}}$ at the origin, we can apply Proposition 3.1 (cf. Remark 3.2): there exist an open neighborhood of the origin $W \subset V_{1}$ and $v \in G^{s}(W)$ satisfying

$$
\mathrm{Q}_{s, r}\left(\mathrm{X}, \mathrm{L}^{\sharp}\right) v=\mathrm{Q}_{s, r}\left(\mathrm{X}, \mathrm{L}^{\sharp}\right) u \quad \text { in } W .
$$

By Proposition 3.2 (c. Remark 3.4) applied to $u-v$ substituted for $v$ and taking into account that $v \in G^{s}(W)$, the existence of a neighborhood of the origin $W_{0} \subset W$ follows such that $u \in G^{s}\left(W_{0}\right)$.

\section{REFERENCES}

[BCH] S. Berhanu, P.D. Cordaro and J. Hounie, An Introduction to Involutive Structures. Cambridge University Press, 2008. MR 2397326

[C] P.A.S. Caetano, Classes de Gevrey em estruturas hipo-analíticas. Ph.D. thesis, University of São Paulo, Brazil, 2001.

[Co] P.D. Cordaro, Representation of hyperfunction solutions in a hypo-analytic structure. Math. Z., 233 (2000), 633-654. MR1759265 (2001f:35070)

$[\mathrm{H}] \quad$ L. Hörmander, Propagation of singularities and semi-global existence theorems for (pseudo)differential operators of principal type. Ann. of Math. (2) 108 (1978), 569-609. MR:512434 (81j:35110)

[Ka] A. Kaneko, Representation of hyperfunctions as measures and some of its applications. J. Fac. Sci. Univ. Tokyo, Sect. IA, 19 (1972), 321-352. MR0336328 (49:1103)

[Ko] H. Komatsu, Ultradistributions, I: Structure theorems and a characterization. J. Fac. Sci. Univ. Tokyo, Sect. IA, 20 (1973), 25-105. MR0320743 (47:9277)

[LL] B. Lascar and N. Lerner, Propagation de singularités Gevrey pour l'équation de CauchyRiemann dégénérée. Israel J. Math. 124 (2001), 299-312. MR.1856522 (2002i:35003)

[Mi] V. Michel, Résolution locale du $\bar{\partial}$ avec régularité Gevrey au bord d'un domaine $r$-convexe. Math. Z., 218 (1995), 305-317. MR1318162 (95m:32027)

[NT] L. Nirenberg and F. Treves, Solvability of a first order linear partial diferential equation. Comm. Pure Appl. Math. 16 (1963), 331-351. MR0163045(29:348)

[Ro] L. Rodino, Linear Partial Differential Operators in Gevrey Spaces. World Scientific Publishing Co., Singapore, 1993. MR.1249275 (95c:35001) 
[T1] F. Treves, Integral representation of solutions of first-order linear partial differential equations, I. Ann. Scuola Norm. Sup. Pisa, Serie IV III, no. 1 (1976), 1-35. MR0399635 $(53: 3478)$

[T2] F. Treves, Hypo-analytic structures (local theory). Princeton University Press, 1992. MR.1200459 (94e:35014)

Department of Mathematics, Universidade Federal de São Carlos, São Carlos, SP, BRAZIL

E-mail address: caetano@dm.ufscar.br

Department of Mathematics, Universidade de São Paulo, São Paulo, SP, Brazil

E-mail address: cordaro@ime.usp.br 